\title{
Orientationally Ordered Silicon Nanocrystal Cuboctahedra in Superlattices
}

Yixuan Yu, ${ }^{\dagger}$ Xiaotang Lu, $^{\dagger}$ Adrien Guillaussier, ${ }^{\dagger}$ Vikas Reddy Voggu, $^{\dagger}$ William Pineros, ${ }^{\dagger}$ Maria de la Mata, ${ }^{\S}$ Jordi Arbiol, ${ }^{\S}, \|_{\odot}$ Detlef-M. Smilgies, ${ }^{\ddagger}$ Thomas M. Truskett, ${ }^{\dagger}$ and Brian A. Korgel ${ }^{*},^{\dagger}(0)$

${ }^{\dagger}$ McKetta Department of Chemical Engineering and Texas Materials Institute, The University of Texas at Austin, Austin, Texas 78712-1062, United States

${ }^{\ddagger}$ Cornell High Energy Synchrotron Source (CHESS), Cornell University, Ithaca, New York 14853, United States

${ }^{\S}$ Catalan Institute of Nanoscience and Nanotechnology (ICN2), CSIC and The Barcelona Institute of Science and Technology (BIST), Campus UAB, Bellaterra, 08193 Barcelona, Catalonia, Spain

"ICREA, Pg. Lluís Companys 23, 08010 Barcelona, Catalonia, Spain

Supporting Information

ABSTRACT: Uniform silicon nanocrystals were synthesized with cuboctahedral shape and passivated with 1-dodecene capping ligands. Transmission electron microscopy, electron diffraction, and grazing incidence wide-angle and small-angle $\mathrm{X}$ ray scattering show that these soft cuboctahedra assemble into face-centered cubic superlattices with orientational order. The preferred nanocrystal orientation was found to depend on the orientation of the superlattices on the substrate, indicating that the interactions with the substrate and assembly kinetics can influence the orientation of faceted nanocrystals in superlattices.

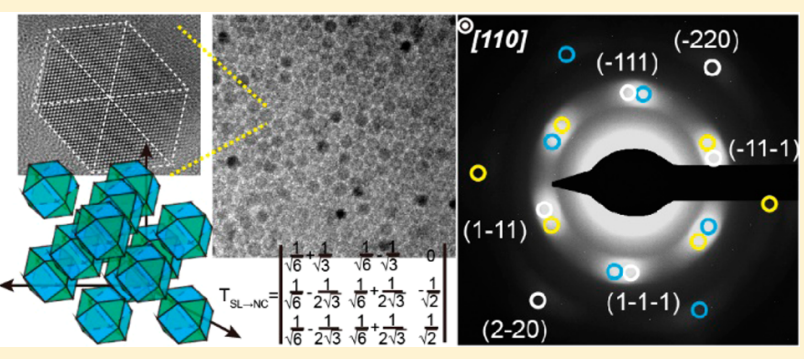

KEYWORDS: Orientational order, silicon nanocrystals, superlattices, transformation matrix

$\mathrm{N}$ anocrystal assemblies are being explored for a variety of applications due in large part to their tunable properties that depend on both the individual nanocrystals and their arrangement. ${ }^{1}$ Some nanocrystals can even be made with highly controlled polyhedral, or faceted, shape and assembled into superlattices with orientational order and coherent crystal structure throughout the superlattice. ${ }^{2-22}$ For silicon (Si), it is now possible to synthesize highly uniform nanocrystals and assemble them into superlattices. ${ }^{23,24} \mathrm{Si}$ nanocrystals have also been made with a faceted cube and tetrahedal shape, albeit not with sufficient uniformity to form superlattices. ${ }^{25-28}$ Here, we demonstrate a synthetic approach that yields organic ligandstabilized Si nanocrystals with uniform cuboctahedral shape that can be assembled into face-centered cubic (fcc) superlattices with preferential orientational order.

Transmission electron microscopy (TEM), electron diffraction, and grazing incidence small angle (GISAXS) and wideangle X-ray scattering (GIWAXS) data show that the superlattices of $\mathrm{Si}$ nanocrystal cuboctahedra exhibit a high degree of orientational order in the fcc superlattices. Furthermore, the relative orientations of the nanocrystals in the fcc superlattices varied with the orientation of the superlattice on the substrate, indicating that not only are packing constraints important, but the substrate and kinetic affects also influence the relative orientation of the nanocrystals.

Figure 1 shows TEM images of several Si nanocrystals with cuboctahedral shape. TEM images provide two-dimensional projections of the nanocrystal shape, so nanocrystals must be imaged with various orientations on the substrate to determine accurately their three-dimensional shape. Figure 1 shows nanocrystals imaged with four different orientations on the substrate and all compare well to those expected for cuboctahedra. For reference, Figure 1a illustrates the shape of a cuboctahedron and how a cuboctahedron would appear with three different orientations on the substrate. The cuboctahedron is a polyhedron with 8 triangular faces, 6 square faces, 12 identical vertices, and 24 identical edges. The cuboctahedron and the diamond cubic Si lattice both have the same rotational symmetry (rotation group 432), each with 24 rotational permutations. $^{29}$ The square and triangular faces of the cuboctahedra are $\{100\}$ and $\{111\}$ planes of diamond cubic Si. Figure 1 shows TEM images of several Si nanocrystals imaged with four different types of orientations on the substrate. The four different orthogonal projections shown in Figure 1 all correspond to nanocrystals with a cuboctahedral shape, as shown in Figure $1 \mathrm{~b}-\mathrm{j}$. The experimentally observed TEM images are also consistent with the HRTEM image simulations shown in Figure 1a. Three-dimensional atomic models of cuboctahedral Si nanocrystals were created using the Rhodius software package. ${ }^{30,31}$ Each cuboctahedral model has

Received: September 25, 2016

Revised: November 10, 2016

Published: November 16, 2016 

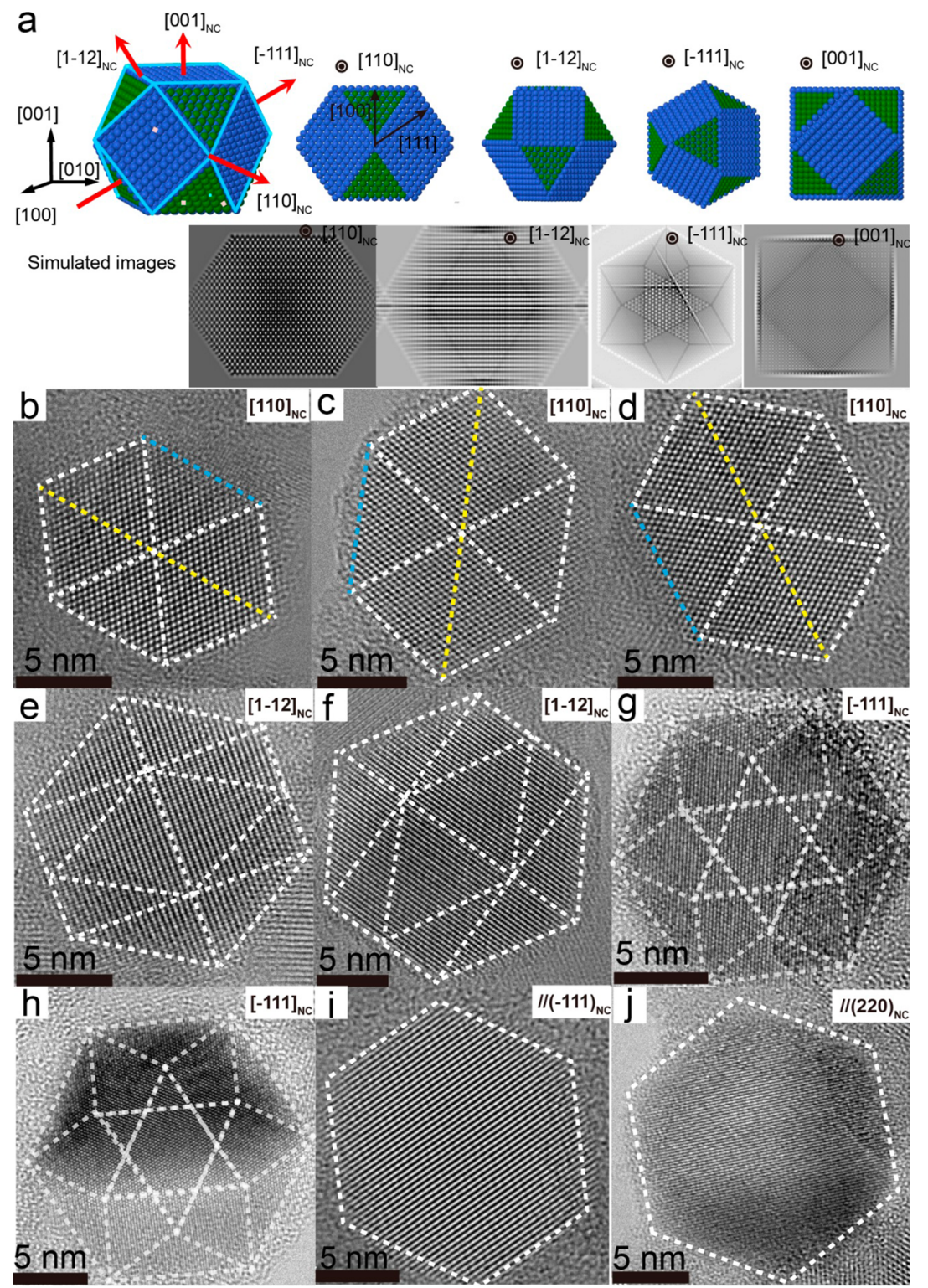

Figure 1. (a) Three-dimensional atomic models of a Si nanocrystal with cuboctahedral shape. The blue square facets are $\{100\}_{\mathrm{NC}}$ planes and the green triangular facets are $\{111\}_{\mathrm{NC}}$ planes. Four different two-dimensional projections and their simulated TEM images are also shown. (b-j) TEM images of Si nanocrystals with four different orientations. $(\mathrm{b}-\mathrm{d})$ The vertex projections, corresponding to the $\langle 110\rangle_{\mathrm{NC}}$ zone axes; $(\mathrm{e}, \mathrm{f})$ edge projections, corresponding to the $\langle 112\rangle_{\mathrm{NC}}$ zone axes; $(\mathrm{g}, \mathrm{h})$ triangular face projections, corresponding to the $\langle 111\rangle_{\mathrm{NC}}$ zone axes; (i,j) skewed projections that show the $\{111\}$ and $\{220\}$ lattice fringes of Si. The labels " $/ /(h k l)_{\mathrm{NC}}$ " indicate that the electron beam is oriented parallel to an $(h k l)_{\mathrm{NC}}$ crystal plane.

14 different facets belonging to the $\{111\}$ and $\{002\}$ family planes, 8 and 6 facets, respectively. A basic model with 6680 atoms was used to illustrate the nanocrystal morphology along different orientations (Figure la (top)), while a more complex model with similar size to the experimentally characterized nanocrystals (about 53360 atoms) was used for HRTEM image simulations (Figure 1a (bottom)). For the HRTEM image simulations, parameters were used equivalent to those corresponding to the JEOL 2010F TEM used for the experimental imaging: acceleration voltage $=200 \mathrm{keV}, \mathrm{Cc}=$ $1.1 \mathrm{~mm}, \mathrm{Cs}=0.5 \mathrm{~mm}$, energy spread $=0.80 \mathrm{eV}$, defocus $=53.4$, $73.4,43.4$, and $53.4 \mathrm{~nm}$ for the [110], [112], [-111] and [001] projections, respectively. TEM images of nanocrystals with $[001]_{\mathrm{NC}}$ zone axis were not observed, probably due to the low lattice fringe contrast at this projection, as shown in the simulated TEM image. In addition, the [001] projection contains the $\{220\}$ planes, which have $d$-spacing $(0.19 \mathrm{~nm})$ near the limit of the resolution of the TEM used for the imaging.

The Si nanocrystals with cuboctahedral shape were synthesized by heating hydrogen silsesquioxane (HSQ) while cycling the temperature between 800 and $1400{ }^{\circ} \mathrm{C}$. (See Supporting Information for Experimental Details.) This process yields $\mathrm{Si}$ nanocrystals embedded in an $\mathrm{SiO}_{2}$ matrix and the cuboctahedral shape results from the relative $\mathrm{Si} / \mathrm{SiO}_{2}$ interface energies of the $\{100\}$ and $\{111\}$ facets of Si. The $\{100\}$ surface has the lowest $\mathrm{Si} / \mathrm{SiO}_{2}$ interface energy and the $\{111\}$ facets have the next lowest energy. ${ }^{32,33}$ The $\{110\}$ facets have significantly higher energy due to their high in-plane $\mathrm{Si}-\mathrm{Si}$ bond density. ${ }^{34}$ The two lowest energy facets of $\{111\}$ and $\{100\}$ present themselves during nanocrystal growth and lead to the cuboctahedral shape that is observed. ${ }^{35}$ After heating 
HSQ the nanocrystals are liberated from the oxide by etching with hydrofluoric acid and then passivated by thermal hydrosilylation with 1-dodecene. A size selective precipitation is used to finally narrow the uniformity of the nanocrystals.

The nanocrystals are of course still not perfectly uniform after size selection. The remaining statistical variation in nanocrystal size and shape includes a distribution in the number of atoms in the nanocrystal core and varying edge and corner truncations. (See Figures S8 and S9 in Supporting Information for examples.) Another source of variation in the nanocrystal shape are crystallographic defects, which distort the cuboctahedral shape. For example, Figure 2 compares a

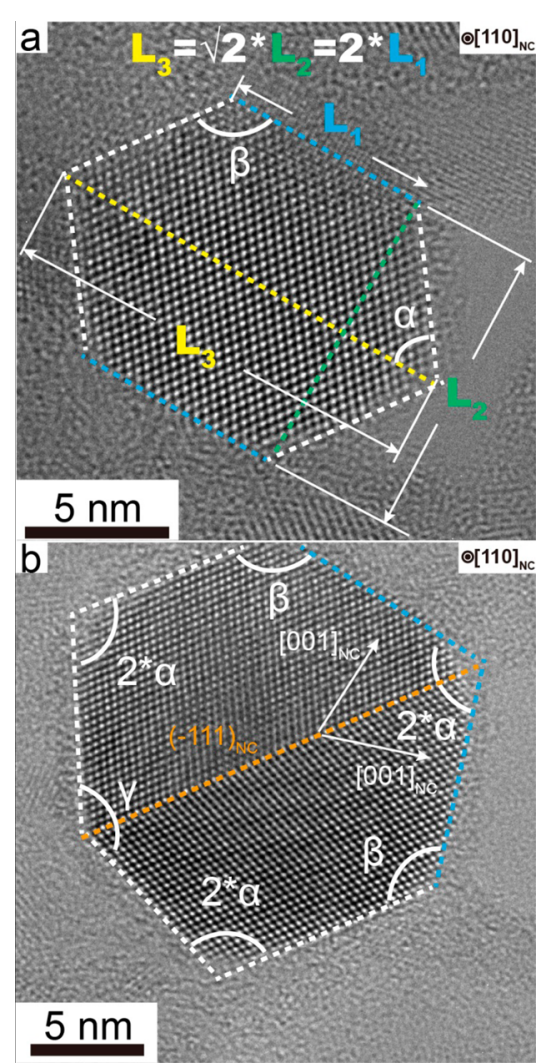

Figure 2. High-resolution TEM images of faceted Si nanocrystals imaged down the $[110]_{\mathrm{NC}}$ zone axis. The image in (a) matches a vertex projection of a perfect cuboctahdron with angles between the facets of $\alpha=54.7^{\circ}, \beta=125.3^{\circ}$ and the expected relationships between the corner-to-corner and face-to-face separations and edge lengths. The image in (b) shows a nanocrystal with a $(-111)_{\mathrm{NC}}$ twin. Twinning leads to a distortion from the cuboctahedral shape with $[110]_{\mathrm{NC}}$ zone axis showing a distorted hexagonal projection with three angles between facets of $2 \alpha=109.5^{\circ}, \beta=125.3^{\circ}$, and $\gamma=141.1^{\circ}$.

nanocrystal with a perfect cuboctahedral shape and a nanocrystal with a shape distorted by a twin. In Figure $2 \mathrm{a}$, a vertex projection is shown of the nanocrystal with cuboctahedral shape (i.e., imaged down the $[110]_{\mathrm{NC}}$ zone axis) having the characteristic distorted hexagonal shape with angles of $2 \alpha=$ $109.5^{\circ}$ and $\beta=125.3^{\circ}$ between facets. The longest corner-tocorner distance is $\sqrt{ } 2$ times longer than the distance between square $\{100\}$ facets and twice as long as the cuboctahedron edge length, as expected for a cuboctahedron. The nanocrystal in Figure $2 \mathrm{~b}$ has a $(-111)_{\mathrm{NC}}$ twin. The twin defect leads to a distorted shape with three angles $2 \alpha=109.5^{\circ}, \beta=125.3^{\circ}$, and $\gamma$ $=141.1^{\circ}$ between the facets in the projection.
Figures 3 and 4 show TEM images and GISAXS data obtained from superlattices of the cuboctahedral Si nanocrystals. The superlattices have fcc structure. The TEM images in Figure $3 a-d$ show superlattices with four different orientations on the substrate. The fast Fourier transforms (FFTs) of the TEM images in Figure $3 a-d$ are shown in Figure $3 e-h$ and all indexed to fcc superlattices, revealing the orientation of the superlattice on the substrate. $(111)_{\text {SL }}$ projections were most commonly observed. Typically, about $70 \%$ of the grid are covered by superlattice with $(111)_{\mathrm{SL}}$ planes being exposed, while $(-211)_{\mathrm{SL}},(110)_{\mathrm{SL}}$, and $(001)_{\mathrm{SL}}$ superlattice projections can also be observed from approximately $10 \%, 5 \%$, and $5 \%$ of the total area, respectively. Approximately $10 \%$ of the sample on a TEM grid would be a disordered nanocrystal ensemble. The GISAXS pattern indicates that the fcc superlattice is oriented with $(111)_{\mathrm{SL}}$ planes on the substrate and the lattice constant of the superlattices is $a_{\mathrm{SL}, \mathrm{fcc}}=16.6 \mathrm{~nm}$.

Electron diffraction and GIWAXS both probe the atomic Si lattice of the nanocrystals. Both electron diffraction and GIWAXS data show that the nanocrystals have a preferred crystallographic orientation. For example, Figure 3i-1 shows the electron diffraction patterns obtained from the superlattices imaged in Figure $3 \mathrm{a}-\mathrm{d}$. The diffraction spots (as opposed to rings) indicate that the nanocrystals have a preferred crystallographic orientation in the superlattice. The appearance of spots in the GIWAXS pattern in Figure $4 \mathrm{~b}$ as well indicates that the preferential orientation of the atomic $S i$ lattice in the superlattice is relatively long-range. Both the electron diffraction patterns and the GIWAXS patterns index to diamond cubic Si. In addition, the GIWAXS data indicate that the atomic $(110)_{\mathrm{NC}}$ planes are predominantly oriented parallel to the substrate, which is consistent with the TEM and electron diffraction data as well, as discussed in further detail below.

The relative orientation of the Si nanocrystal cuboctahedra in the fcc superlattice can be determined by analyzing the electron diffraction patterns in relation to the superlattice orientation observed by TEM. For instance, Figure $3 \mathrm{a}$ is a projection of the $(111)_{S L}$ superlattice plane. The electron diffraction pattern in Figure $3 \mathrm{i}$ was obtained from this superlattice region. It exhibits six spots with hexagonal symmetry. The $d$-spacing obtained from the diffraction pattern matches the $(111)_{\mathrm{NC}}$ atomic Si planes. If the sample were a single crystal of $\mathrm{Si}$, there would only be four $\{111\}_{\mathrm{NC}}$ diffraction spots, not six, and the diffraction pattern is actually an overlay of three different spot patterns. These three spot patterns are indicated by white, yellow, and blue circles. They arise from diffraction obtained down the same $[110]_{\mathrm{NC}}$ zone axis of the $\mathrm{Si}$ atomic lattice from nanocrystals with three different rotational orientations. (See Supporting Information for complete indexing of the electron diffraction patterns and a discussion about the analysis.)

Figure $3 \mathrm{j}$ shows an electron diffraction pattern obtained from a superlattice with a different orientation. The superlattice is imaged in the $[-211]_{\mathrm{SL}}$ direction, and the electron diffraction pattern consists of an overlay of two spot patterns corresponding to nanocrystals imaged down the $[-110]_{\mathrm{NC}}$ (white circles) and $[1-12]_{\mathrm{NC}}$ zone axes (yellow circles). The superlattice in Figure $3 \mathrm{c}$ is imaged in the $[110]_{\mathrm{SL}}$ direction and the electron diffraction pattern obtained from this region (Figure 3k) corresponds to two sets of diffraction spots obtained from nanocrystals oriented with either $[1-12]_{\mathrm{NC}}$ (white circles) or $[-111]_{\mathrm{NC}}$ (yellow circles) zone axes parallel to the beam. The superlattice in Figure $3 \mathrm{~d}$ is imaged down the 


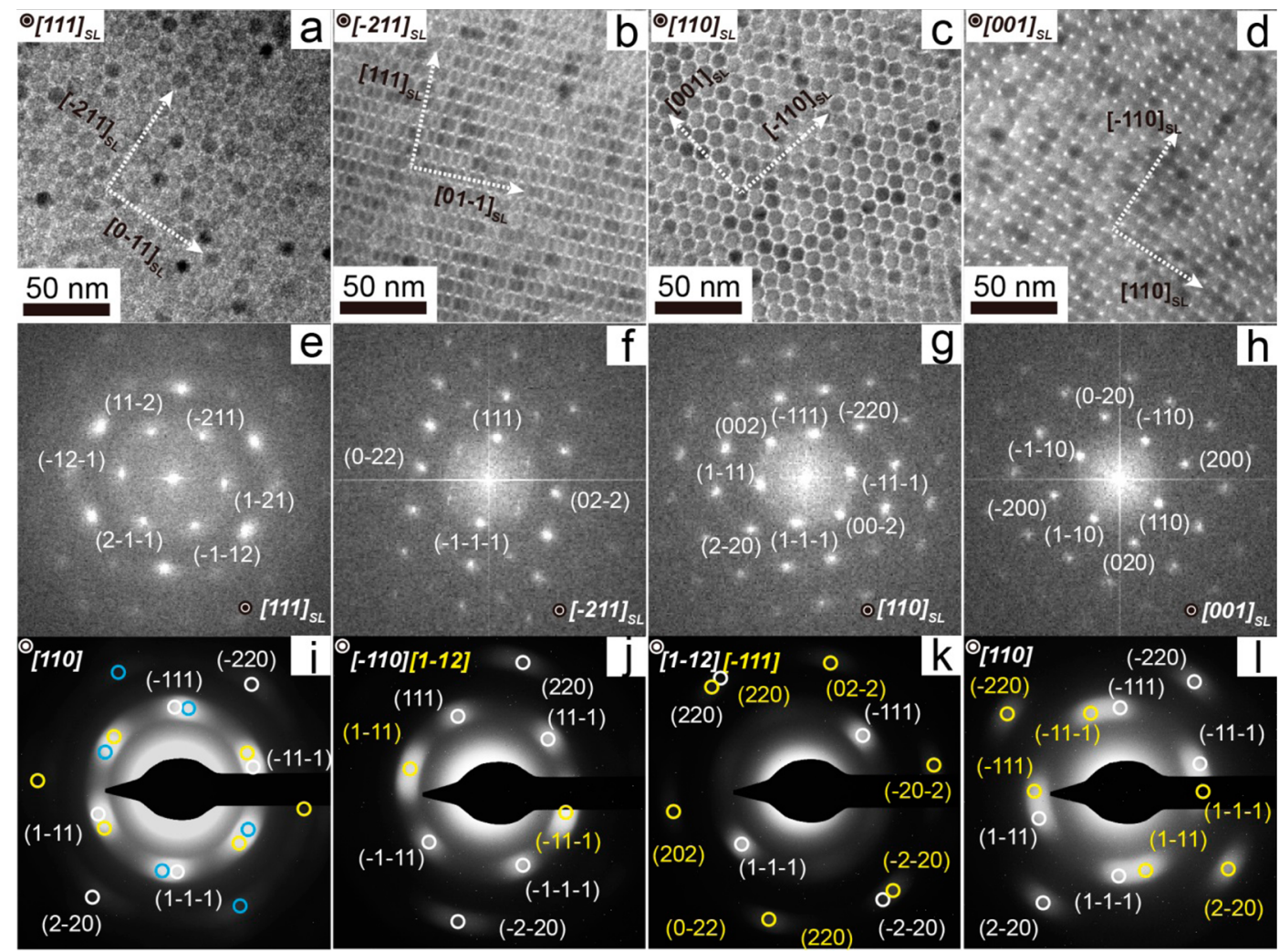

Figure 3. TEM images of superlattices formed by $\mathrm{Si}$ nanocrystals with cuboctahedral shape. The superlattices have fcc structure with various orientations with (a) $(111)_{S \mathrm{SL}}$ (b) $(-211)_{\mathrm{SL}}$ (c) $(110)_{\mathrm{SL}}$, and (d) $(001)_{\mathrm{SL}}$ planes parallel to the substrate, corresponding to FFT in (e-h). (i-l) Electron diffraction patterns taken from $\sim 1 \mu \mathrm{m}^{2}$ area of superlattice shown in $(\mathrm{a}-\mathrm{d})$, respectively.

$[001]_{\mathrm{SL}}$ zone axis and the electron diffraction pattern of this region shown in Figure 31 is an overlay of two different spot patterns corresponding to the $[110]_{\mathrm{NC}}$ zone axis from nanocrystals with two different orientations.

From the TEM and electron diffraction data, the preferred orientations of the cuboctahedra in the superlattice can be determined. A cuboctahedron and an fcc lattice both have the same rotational symmetry (rotation group 432) with 24 rotational permutations. ${ }^{29}$ So, there are 24 possible rotational orientations of the cuboctahedral nanocrystals in each superlattice position. The orientational relationship between the superlattice and the $\mathrm{Si}$ atomic lattice of the nanocrystals is described by a transformation matrix, $T_{\mathrm{SL} \rightarrow \mathrm{NC}}$. $T_{\mathrm{SL} \rightarrow \mathrm{NC}}$ provides the relationship between the superlattice orientation and the atomic Si lattice orientation: $\left[h^{\prime} k^{\prime} l^{\prime}\right]_{\mathrm{NC}}=[h k l]_{\mathrm{SL}} \cdot T_{\mathrm{SL} \rightarrow \mathrm{NC}}$, in which $[h k l]_{\mathrm{SL}}$ is any given direction in the superlattice, and $\left[h^{\prime} k^{\prime} l^{\prime}\right]_{\mathrm{NC}}$ is the direction in the atomic lattice parallel to $[h k l]_{\mathrm{SL}} \cdot T_{\mathrm{SL} \rightarrow \mathrm{NC}}$ is determined from the crystal orientations observed by electron diffraction. For example, the TEM and electron diffraction data in Figure 3a,i show that the $[111]_{\mathrm{SL}}$, $[-211]_{\mathrm{SL}}$, and $[0-11]_{\mathrm{SL}}$ directions in the superlattice are oriented parallel to the $[220]_{\mathrm{NC}},[-220]_{\mathrm{NC}}$, and $[002]_{\mathrm{NC}}$ directions of the atomic $\mathrm{Si}$ lattice in the nanocrystals, respectively, such that

$$
\begin{aligned}
& \frac{1}{\sqrt{3}}[111]_{\mathrm{SL}} \cdot T_{\mathrm{SL} \rightarrow \mathrm{NC}}=\frac{1}{\sqrt{2}}[110]_{\mathrm{NC}} \\
& \frac{1}{\sqrt{6}}[-211]_{\mathrm{SL}} \cdot T_{\mathrm{SL} \rightarrow \mathrm{NC}}=\frac{1}{\sqrt{2}}[-110]_{\mathrm{NC}}
\end{aligned}
$$

$$
\frac{1}{\sqrt{2}}[0-11]_{\mathrm{SL}} \cdot T_{\mathrm{SL} \rightarrow \mathrm{NC}}=[001]_{\mathrm{NC}}
$$

which gives

$$
T_{\mathrm{SL} \rightarrow \mathrm{NC}}=\left|\begin{array}{lll}
\frac{1}{\sqrt{6}}+\frac{1}{\sqrt{3}} & \frac{1}{\sqrt{6}}-\frac{1}{\sqrt{3}} & 0 \\
\frac{1}{\sqrt{6}}-\frac{1}{2 \sqrt{3}} & \frac{1}{\sqrt{6}}+\frac{1}{2 \sqrt{3}} & -\frac{1}{\sqrt{2}} \\
\frac{1}{\sqrt{6}}-\frac{1}{2 \sqrt{3}} & \frac{1}{\sqrt{6}}+\frac{1}{2 \sqrt{3}} & \frac{1}{\sqrt{2}}
\end{array}\right|
$$

This transformation matrix corresponds to the Si nanocrystals with the specific orientation that gave rise to the electron diffraction that is labeled with white circles in Figure 3i. This is one of the 24 rotational permutations. Rotational permutations of a cubic lattice, that is, fcc, are related by common rotational matrices, $A_{i}, i=1,2, \ldots 24$. For instance, $A=\left|\begin{array}{ccc}-1 & 0 & 0 \\ 0 & -1 & 0 \\ 0 & 0 & 1\end{array}\right|$ describes a rotation of $180^{\circ}$ around the $[001]_{\mathrm{SL}}$ axis, which turns $[100]_{\mathrm{SL}}$ into $[-100]_{\mathrm{SL}},[010]_{\mathrm{SL}}$ into $[0-10]_{\mathrm{SL}}$, and $[001]_{\mathrm{SL}}$ into $[001]_{\mathrm{SL}}$. The transformation matrices for the other nanocrystal orientations are

$$
T_{i}=A_{i} \cdot T_{\mathrm{SL} \rightarrow \mathrm{NC}}, \quad i=1,2, \ldots, 24
$$

Hence, the electron diffraction patterns obtained from a superlattice imaged down the $[111]_{\mathrm{SL}}$ zone axis can be indexed by considering only six orientations of the nanocrystals, and the spot patterns of three of the six orientations overlap; for example, diffraction from the $[110]_{\mathrm{NC}}$ zone axis overlaps with 


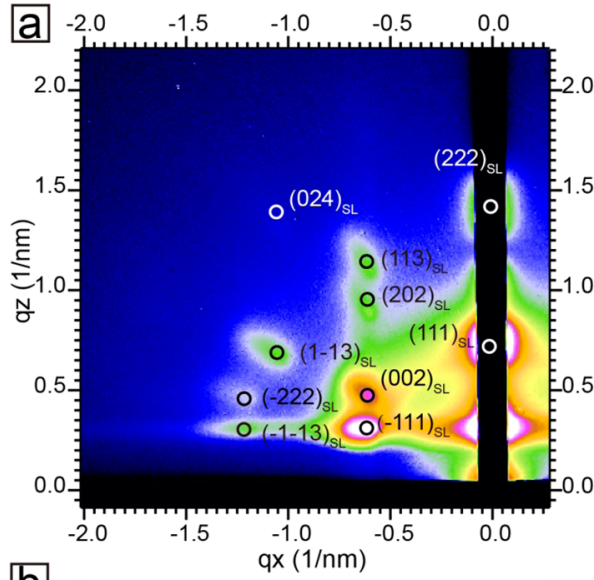

Once $T_{\mathrm{SL} \rightarrow \mathrm{NC}}$ is known, the electron diffraction patterns can be predicted for other superlattice orientations. For example, superlattices imaged down the $[-211]_{\mathrm{SL}}$ zone axis should give rise to diffraction spots corresponding to observation of the Si atomic lattice down the $[-110]_{\mathrm{NC}}$ zone axis (white circles in Figure $3 \mathrm{j}$ ), since

$$
[-211]_{\mathrm{SL}} \cdot T_{\mathrm{SL} \rightarrow \mathrm{NC}}=\sqrt{3}[-110]_{\mathrm{NC}}
$$

In Figure 3j, some additional diffraction spots corresponding to the $[1-12]_{\mathrm{NC}}$ zone axis (yellow circles) are also observed that are not predicted by $T_{\mathrm{SL} \rightarrow \mathrm{NC}}$. This is because some nanocrystals are oriented preferentially with a $[1-1 \sqrt{ } 6]_{\mathrm{NC}}$ zone axis,

$$
[-211]_{\mathrm{SL}} \cdot\left|\begin{array}{ccc}
0 & 0 & 1 \\
1 & 0 & 0 \\
0 & 1 & 0
\end{array}\right| \cdot T_{\mathrm{SL}-\text { to-NC}}=\frac{\sqrt{3}}{2}[1-1 \sqrt{6}]_{\mathrm{NC}}
$$

Nanocrystals oriented on a $[1-1 \sqrt{ } 6]_{\mathrm{NC}}$ zone axis do not give rise to diffraction spots, but there is enough rotational disorder of the nanocrystals in the superlattice such that some will lie on a $[1-12]_{\mathrm{NC}}$ zone axis, an orientation that does give rise to spots. The angle between the $[1-1 \sqrt{ } 6]_{\mathrm{NC}}$ and $[1-$ $12]_{\mathrm{NC}}$ zone axes is only $5.8^{\circ}$. The rotational disorder in the superlattice is discussed in more detail below. Additionally, there are four possible nanocrystal orientations with a [1$12]_{\mathrm{NC}}$ zone axis, compared to only two nanocrystal orientations with a $[-110]_{\mathrm{NC}}$ zone axis, which influences the spot pattern.

Calculation of $T_{\mathrm{SL} \rightarrow \mathrm{NC}}$ for the other two superlattice projections, that is, with $(110)_{\mathrm{SL}}$ (Figure $3 \mathrm{k}$ ) and $(001)_{\mathrm{SL}}$ (Figure 31) orientations, reveal that the nanocrystals have different relative orientations than in the superlattices observed with $(111)_{\mathrm{SL}}$ orientation. The transformation matrices describing the relative orientations, $T_{\mathrm{SL} \rightarrow \mathrm{NC}(110)}$ and $T_{\mathrm{SL} \rightarrow \mathrm{NC}(001)}$ calculated from the TEM and electron diffraction data in Figure 3 are

diffraction from the $[-1-10]_{\mathrm{SL}}$ zone axis. A complete list of common rotational matrices and a detailed indexing of the diffraction patterns is described in Supporting Information.

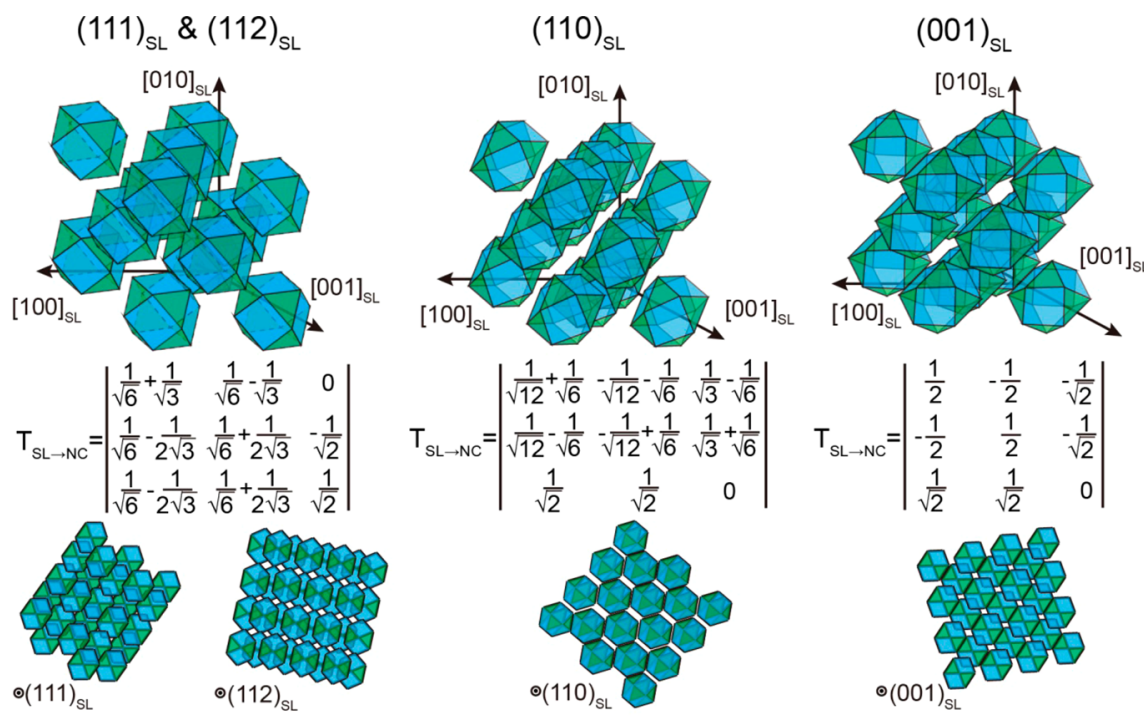

Figure 5. Illustration of the relative orientations of Si nanocrystals with cuboctahedral shape in fcc superlattices that were determined experimentally by TEM and electron diffraction, as in Figure 3 for example. The corresponding transformation matrices are also shown. 


$$
\begin{aligned}
& T_{\mathrm{SL} \rightarrow \mathrm{NC}(110)} \\
& =\left|\begin{array}{ccc}
\frac{1}{\sqrt{12}}+\frac{1}{\sqrt{6}} & -\frac{1}{\sqrt{12}}-\frac{1}{\sqrt{6}} & \frac{1}{\sqrt{3}}-\frac{1}{\sqrt{6}} \\
\frac{1}{\sqrt{12}}-\frac{1}{\sqrt{6}} & -\frac{1}{\sqrt{12}}+\frac{1}{\sqrt{6}} & \frac{1}{\sqrt{3}}+\frac{1}{\sqrt{6}} \\
T_{\mathrm{SL} \rightarrow \mathrm{NC}(001)} & =\left|\begin{array}{ccc}
\frac{1}{\sqrt{2}} & 0 \\
-\frac{1}{2} & \frac{1}{2} & -\frac{\sqrt{2}}{2} \\
\frac{\sqrt{2}}{2} & \frac{\sqrt{2}}{2} & 0
\end{array}\right|
\end{array}\right|
\end{aligned}
$$

The corresponding orientations of the cuboctahedra in the fcc superlattice positions are illustrated in Figure 5. The nanocrystal orientations observed by TEM for the nanocrystals with $(111)_{\text {SL }}$ planes oriented on the substrates are consistent with the superlattices examined by GISAXS/GIWAXS.

The different nanocrystal orientations for the superlattice orientations observed by TEM might be the result of a kinetic effect in which the formation process of the superlattices influences the orientation of the nanocrystals, or an interaction between the nanocrystal shape and the substrate. Thapar and Escobedo $^{36}$ have recently shown that hard polyhedra can exhibit significantly different superlattice nucleation kinetics than hard spheres for example. Interactions with the substrate might also influence the orientation of the nanocrystals. It appears that the cuboctahedral Si nanocrystals prefer to lie on the substrate on a vertex. The dodecene-capped Si nanocrystals are hydrophobic and the substrates, either an amorphous carbon film or native $\mathrm{Si}$ oxide, are slightly hydrophilic. The interactions between the substrate and the nanocrystals will depend on the superlattice orientation. For example, if a superlattice with a $(0-11)_{\text {SL }}$ plane parallel to the substrate had nanocrystals with the same orientation as those with $(111)_{\mathrm{SL}}$ planes on the substrate, the nanocrystals would have the $[001]_{\mathrm{NC}}$ direction perpendicular to the substrate, which would be unfavorable as it would require cuboctahedra to contact the substrate with square $(001)_{\mathrm{NC}}$ facets.

The amount of orientational disorder in the superlattice can be determined from the breadth of the electron diffraction spots. Figure 6 shows an electron diffraction pattern obtained from a region of only a very few nanocrystals. This pattern consists of a collection of sharp spots, as opposed to the diffuse spots shown in Figure $3 \mathrm{i}-1$, which were obtained from a much larger region of nanocrystals. The breadth of each diffraction spot provides a measure of the rotational distribution of nanocrystals in the superlattice. As shown in Figure 6a, the collection of spots has a breadth of $\pi / 6$. This one "diffraction spot" corresponds to four specific nanocrystal orientations (Figure S5), and a rotational freedom of $\Delta \omega=\pi / 6^{*} \sqrt{1 / 4}=\pi / 12$. This is an estimate of the variation in rotational ordering of the cuboctahedra in the superlattice.

The Wigner-Seitz (W-S) cell of fcc lattice has a rhombic dodecahedral shape. We have recently proposed that the space filling of nanocrystals in the W-S cell of a superlattice can

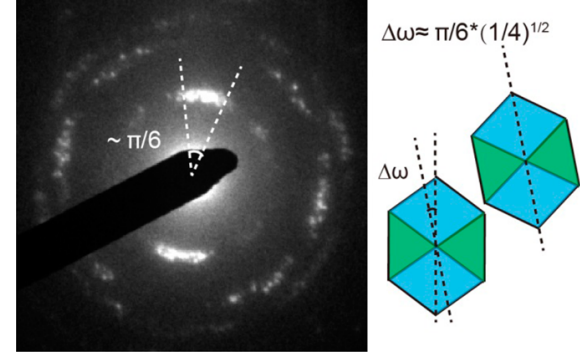

Figure 6. Electron diffraction pattern obtained from a region of about $100 \mathrm{Si}$ nanocrystals, showing how the diffuse diffraction spots evolve from a collection of sharp diffraction spots from individual nanocrystals. The breadth of the diffraction spots reflects the distribution of nanocrystal orientations in the superlattice.

provide insight about the preferred superlattice structure, at least in the case of fcc versus body-centered cubic structure. ${ }^{37}$ Figure 7 illustrates the space-filling of the nanocrystal cuboctahedron in the $\mathrm{W}-\mathrm{S}$ cell for a superlattice with a $(111)_{S L}$ orientation on the substrate. To accommodate the difference in geometry, ligands must provide some ability to flex and bend and fill available space in the superlattice. For example, the fcc superlattice geometry requires the separation between $\{100\}_{\mathrm{NC}}$ facets of neighboring cuboctahedral nano-

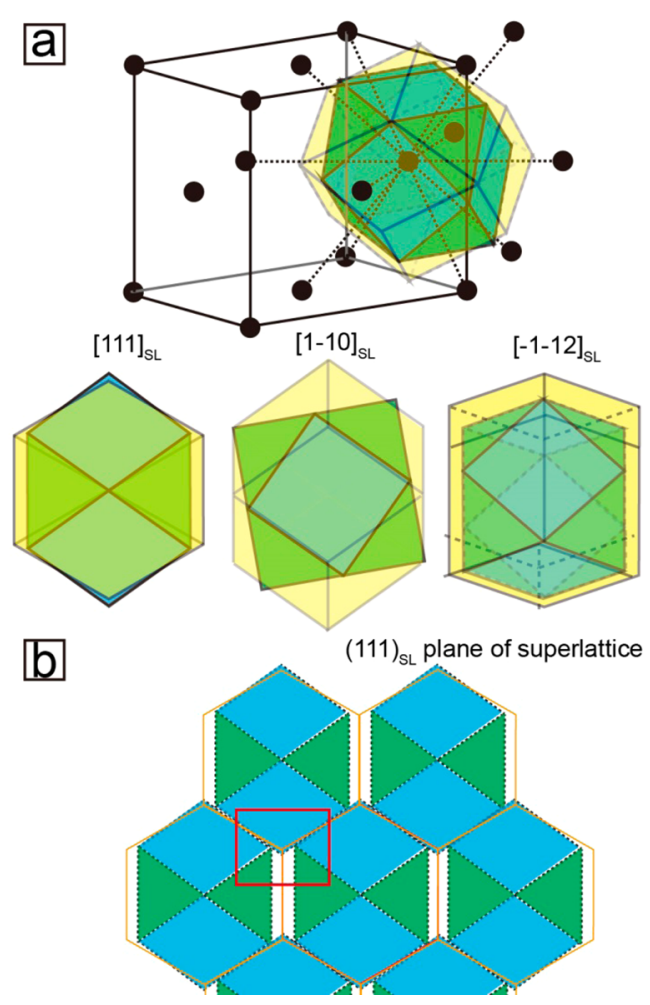

Figure 7. (a) Illustration of a cuboctahedral nanocrystal fit into the rhombic dodecahedron W-S cell of the fcc superlattice. The nanocrystal volume is drawn to include the capping ligands. The nanocrystal orientation drawn is with the $(110)_{\mathrm{NC}}$ atomic Si plane oriented parallel with the $(111)_{\text {SL }}$ plane. Projections of three different directions are shown. (b) A (111) sL projection of the superlattice, in which the orange hexagons are the $\mathrm{W}$-S cells, shows that the small volume of Si nanocrystal ligand layer that protrudes out of the W-S cell can fit into the neighboring W-S cell, as highlighted by the red rectangle. Also, see accompanying videos of the cuboctahedral nanocrystals within the fcc W-S cell in Supporting Information. 
crystals to be larger than that of $\{111\}_{\mathrm{NC}}$ facets, which might be accommodated by the difference of capping ligand density on those two facets. Theoretical models of dense hard cuboctahedra have not predicted the fcc phase with orientational order, ${ }^{38-46}$ which is perhaps not surprising since very dense collections would induce facet-interactions that distort the lattice. The less dense fcc structure with rotational disorder, the "rotator phase", on the other hand, would not exhibit the same space-filling constraints. In our case, we did not observe the rotator phase, but probably because the nanocrystal density is too high. The rotator phase has been observed for $\mathrm{PbS}$ nanocrystal superlattices under a saturated solvent vapor that expands slightly the superlattice from its solvent-free density. ${ }^{47}$ In our case, the W-S cell is too small relative to the nanocrystal size to allow free rotation of the nanocrystals, and the nanocrystals are frozen into a specific orientation. The orientation of the nanocrystals was found to deviate by up to $\pi / 12$ from one of the 24 rotational permutations. Part of this deviation is due to the inhomogeneity in Si nanocrystal size and shape. They are not perfect cuboctahedra due to twinning defects, truncation of corners, rounding of facets, and the soft capping ligand shell. (See Supporting Information for further discussion.)

In conclusion, Si nanocrystals were synthesized with uniform cuboctahedral shape. These nanocrystals assemble into fcc superlattices with orientational order. Theoretical predictions of hard cuboctahedral particles have only predicted for fcc plastic crystals (a rotator phase with no orientational order) or a densely packed tri-interlocking distorted simple cubic structure. ${ }^{38-46}$ The soft ligand shell of the nanocrystals prevents strong facet interactions and provides enough spherical symmetry for the nanocrystals to pack into an fcc structure; however, the anisotropic shape of the nanocrystals is still sufficient to lock the nanocrystals with specific orientations in the superlattice, as the available volume in the W-S cell of the fcc lattice compared to the cuboctahedral shape of the nanocrystals is quite small. No angular lattice distortions from cubic symmetry were observed. The relative orientation of cuboctahedral Si nanocrystals in the fcc superlattice can be described with a transformation matrix, $T_{\mathrm{SL} \rightarrow \mathrm{NC}}$, to help index electron diffraction patterns without ambiguity. The superlattice orientation on the substrate was observed to influence the preferred crystallographic orientation of the nanocrystals. This indicates that the superlattice assembly kinetics and the nanocrystal-substrate interactions influence the preferred orientations of faceted or polyhedral nanocrystals in a superlattice.

\section{ASSOCIATED CONTENT}

\section{S Supporting Information}

The Supporting Information is available free of charge on the ACS Publications website at DOI: 10.1021/acs.nanolett.6b04006.

Experimental details, complete list of common rotational matrices correlating rotational permutations of cubic lattice, detailed analyses and indexing of electron diffraction patterns, high-resolution TEM images showing imperfect cuboctahedral shapes of Si nanocrystals, additional TEM images of fcc superlattices, SEM images of fcc superlattices, and photoluminescence spectra of the cuboctahedral Si nanocrystals (PDF)
The rotation of a cuboctahedral nanocrystal in the fcc $\mathrm{W}-\mathrm{S}$ cell at low densities (AVI)

The rotation of a cuboctahedral nanocrystal in the fcc $\mathrm{W}-\mathrm{S}$ cell at high densities (AVI)

\section{AUTHOR INFORMATION}

\section{Corresponding Author}

*E-mail: korgel@che.utexas.edu. Phone: +1-512-471-5633.

ORCID

Jordi Arbiol: 0000-0002-0695-1726

Brian A. Korgel: 0000-0001-6242-7526

\section{Notes}

The authors declare no competing financial interest.

\section{ACKNOWLEDGMENTS}

This research was funded by the Robert A. Welch Foundation (Grant F-1464 and F-1696) and the National Science Foundation (Grant CBET-1403768). CHESS is supported by the NSF \& NIH/NIGMS via NSF award DMR-1332208. J.A. and M.d.l.M. acknowledge funding from Generalitat de Catalunya 2014 SGR 1638 and MINECO projects MAT2014-59961-C2-2-R and Severo Ochoa Program (Grant SEV-2013-0295).

\section{REFERENCES}

(1) Kovalenko, M. V.; Manna, L.; Cabot, A.; Hens, Z.; Talapin, D. V.; Kagan, C. R.; Klimov, V. I.; Rogach, A. L.; Reiss, P.; Milliron, D. J.; Guyot-Sionnest, P.; Konstantatos, G.; Parak, W. J.; Hyeon, T.; Korgel, B. A.; Murray, C. B.; Heiss, W. ACS Nano 2015, 9, 1012-1057.

(2) Li, R.; Bian, K.; Hanrath, T.; Bassett, W. A.; Wang, Z. J. Am. Chem. Soc. 2014, 136, 12047-12055.

(3) Choi, J. J.; Bealing, C. R.; Bian, K.; Hughes, K. J.; Zhang, W.; Smilgies, D.-M.; Hennig, R. G.; Engstrom, J. R.; Hanrath, T. J. Am. Chem. Soc. 2011, 133, 3131-3138.

(4) Zhang, J.; Luo, Z.; Quan, Z.; Wang, Y.; Kumbhar, A.; Smilgies, D.-M.; Fang, J. Nano Lett. 2011, 11, 2912-2918.

(5) Zhang, J.; Luo, Z.; Martens, B.; Quan, Z.; Kumbhar, A.; Porter, N.; Wang, Y.; Smilgies, D.-M.; Fang, J. J. Am. Chem. Soc. 2012, 134, 14043-14049.

(6) Boneschanscher, M. P.; Evers, W. H.; Geuchies, J. J.; Altantzis, T.; Goris, B.; Rabouw, F. T.; van Rossum, S. A. P.; van der Zant, H. S. J.; Siebbeles, L. D. A.; Van Tendeloo, G.; Swart, I.; Hilhorst, J.; Petukhov, A. V.; Bals, S.; Vanmaekelbergh, D. Science 2014, 344, 1377-1380.

(7) Choi, J. J.; Bian, K.; Baumgardner, W. J.; Smilgies, D.-M.; Hanrath, T. Nano Lett. 2012, 12, 4791-4798.

(8) Henzie, J.; Grünwald, M.; Widmer-Cooper, A.; Geissler, P. L.; Yang, P. Nat. Mater. 2011, 11, 131-137.

(9) Seo, D.; Park, J. C.; Song, H. J. Am. Chem. Soc. 2006, 128, $14863-14870$.

(10) Xia, Y.; Xiong, Y.; Lim, B.; Skrabalak, S. E. Angew. Chem., Int. Ed. 2009, 48, 60-103.

(11) Quan, Z.; Fang, J. Nano Today 2010, 5, 390-411.

(12) Quan, Z.; Siu Loc, W. S.; Lin, C.; Luo, Z.; Yang, K.; Wang, Y.; Wang, H.; Wang, Z.; Fang, J. Nano Lett. 2012, 12, 4409-4413.

(13) Van der Stam, W.; Gantapara, A. P.; Akkerman, Q. A.; Soligno, G.; Meeldijk, J. D.; Van Roij, R.; Dijkstra, M.; de Mello Donega, C. Nano Lett. 2014, 14, 1032-1037.

(14) Mokari, T.; Zhang, M.; Yang, P. J. Am. Chem. Soc. 2007, 129, 9864-9865.

(15) Urban, J. J.; Talapin, D. V.; Shevchenko, E. V.; Murray, C. B. J. Am. Chem. Soc. 2006, 128, 3248-3255.

(16) Gordon, T. R.; Diroll, B. T.; Paik, T.; Doan-Nguyen, V. V. T.; Gaulding, E. A.; Murray, C. B. Chem. Mater. 2015, 27, 2502-2506. 
(17) Sandeep, C. S. S.; Azpiroz, J. M.; Evers, W. H.; Boehme, S. C.; Moreels, I.; Kinge, S.; Siebbeles, L. D. A.; Infante, I.; Houtepen, A. J. ACS Nano 2014, 8, 11499-11511.

(18) Zherebetskyy, D.; Scheele, M.; Zhang, Y.; Bronstein, N.; Thompson, C.; Britt, D.; Salmeron, M.; Alivisatos, P.; Wang, L.-W. Science 2014, 344, 1380-1384.

(19) Boles, M. A.; Talapin, D. V. Science 2014, 344, 1340-1341.

(20) Harfenist, S. A.; Wang, Z. L.; Alvarez, M. M.; Vezmar, I.; Whetten, R. L. J. Phys. Chem. 1996, 100, 13904-13910.

(21) Murray, C. B.; Kagan, C. R.; Bawendi, M. G. Science 1995, 270, $1335-1338$.

(22) Brown, L. O.; Hutchison, J. E. J. Phys. Chem. B 2001, 105, 8911-8916.

(23) Yu, Y.; Bosoy, C. A.; Hessel, C. M.; Smilgies, D.-M.; Korgel, B. A. ChemPhysChem 2013, 14, 84-87.

(24) Yu, Y.; Bosoy, C. A.; Smilgies, D.-M.; Korgel, B. A. J. Phys. Chem. Lett. 2013, 4, 3677-3682.

(25) Yang, Z.; Dobbie, A. R.; Cui, K.; Veinot, J. G. C. J. Am. Chem. Soc. 2012, 134, 13958-13961.

(26) Bapat, A.; Anderson, C.; Perrey, C. R.; Carter, C. B.; Campbell, S. A.; Kortshagen, U. Plasma Phys. Controlled Fusion 2004, 46, B97B109.

(27) Baldwin, R. K.; Pettigrew, K. A.; Garno, J. C.; Power, P. P.; Liu, G.-Y.; Kauzlarich, S. M. J. Am. Chem. Soc. 2002, 124, 1150-1151.

(28) Barrett, C. A.; Dickinson, C.; Ahmed, S.; Hantschel, T.; Arstila, K.; Ryan, K. M. Nanotechnology 2009, 20, 275605.

(29) Conway, J. H.; Burgiel, H.; Goodman-Strauss, C. The Symmetries of Things, 1st ed.; Taylor \& Francis Group: London, 2008.

(30) Bernal, S.; Botana, F. J.; Calvino, J. J.; López-Cartes, C.; PérezOmil, J. A.; Rodrı́guez-Izquierdo, J. M. Ultramicroscopy 1998, 72, 135164.

(31) Arbiol, J.; Cirera, A.; Peiró, F.; Cornet, A.; Morante, J. R.; Delgado, J. J.; Calvino, J. J. Appl. Phys. Lett. 2002, 80, 329-331.

(32) Kong, L.; Lewis, L. J. Phys. Rev. B: Condens. Matter Mater. Phys. 2008, 77, 085204.

(33) Himpsel, F. J.; McFeely, F. R.; Taleb-Ibrahimi, A.; Yarmoff, J. A. Phys. Rev. B: Condens. Matter Mater. Phys. 1988, 38, 6084-6096.

(34) Hesketh, P. J.; Ju, C.; Gowda, S. J. Electrochem. Soc. 1993, 140, $1080-1085$.

(35) Ringe, E.; Van Duyne, R. P.; Marks, L. D. Nano Lett. 2011, 11, 3399-3403.

(36) Thapar, V.; Escobedo, F. A. Phys. Rev. Lett. 2014, 112, 048301.

(37) Goodfellow, B. W.; Yu, Y.; Bosoy, C. A.; Smilgies, D.-M.; Korgel, B. A. J. Phys. Chem. Lett. 2015, 6, 2406-2414.

(38) Damasceno, P. F.; Engel, M.; Glotzer, S. C. Science 2012, 337, 453-457.

(39) Gantapara, A. P.; de Graaf, J.; van Roij, R.; Dijkstra, M. Phys. Rev. Lett. 2013, 111, 015501.

(40) Agarwal, U.; Escobedo, F. A. J. Chem. Phys. 2012, 137, 024905.

(41) Agarwal, U.; Escobedo, F. A. Nat. Mater. 2011, 10, 230-235.

(42) Torquato, S.; Jiao, Y. Phys. Rev. E 2009, 80, 141104.

(43) Volkov, N.; Lyubartsev, A.; Bergström, L. Nanoscale 2012, 4, 4765.

(44) Torquato, S.; Jiao, Y. Nature 2009, 460, 876-880.

(45) Gantapara, A. P.; de Graaf, J.; Van Roij, R.; Dijkstra, M. J. Chem. Phys. 2015, 142, 054904.

(46) Chen, E. R.; Klotsa, D.; Engel, M.; Damasceno, P. F.; Glotzer, S. C. Phys. Rev. X 2014, 4, 011024.

(47) Bian, K.; Choi, J. J.; Kaushik, A.; Clancy, P.; Smilgies, D.-M.; Hanrath, T. ACS Nano 2011, 5, 2815-2823. 